\title{
Awful noises: evaluativism and the affective phenomenology of unpleasant auditory experience
}

\author{
Tom Roberts ${ }^{1}$ (D)
}

Accepted: 30 August 2020/Published online: 17 September 2020

(C) The Author(s) 2020

\begin{abstract}
According to the evaluativist theory of bodily pain, the overall phenomenology of a painful experience is explained by attributing to it two types of representational content-an indicative content that represents bodily damage or disturbance, and an evaluative content that represents that condition as bad for the subject. This paper considers whether evaluativism can offer a suitable explanation of aversive auditory phenomenology - the experience of awful noises - and argues that it can only do so by conceding that auditory evaluative content would be guilty of widespread error. Defending such an error-theory, moreover, comes with several explanatory costs.
\end{abstract}

Keywords Pain $\cdot$ Affective phenomenology $\cdot$ Auditory perception $\cdot$ Sounds

\section{Introduction}

Recent work on the nature of unpleasant mental states has focused closely on the case of somatic pain, and competing theories have been developed in order to explain the aversive phenomenal character of painful experiences ${ }^{1}$ - what it is in virtue of which these states feel bad. Ideally, a philosophical account of pain's unpleasantness would be transferable to other disagreeable sensory states, so as to afford a unified understanding not only of headaches and stubbed toes, but of

\footnotetext{
1 E.g. Aydede (2009), Bain (2013, 2017, 2019), Barlassina and Hayward (2019), Corns (2014, 2018), Cutter and Tye (2011), Gray (2019), Jacobson (2013, 2019) and Klein (2007).

Tom Roberts

tom.roberts@exeter.ac.uk

1 Department of Sociology, Philosophy, and Anthropology, University of Exeter, Rennes Drive, Exeter EX44RJ, UK
} 
encounters with awful noises; noxious tastes, odours, and textures; and-perhapsof nausea, vertigo, claustrophobia, and other embodied discomforts. ${ }^{2}$ The project would be to explain what these undesirable experiences have in common, that sets them apart from affectively-neutral psychological states such as beliefs or visual depictions of everyday scenes.

This paper takes an incremental step in pursuit of this project, by considering unpleasant auditory experiences such as the sound of fingernails being dragged down a chalkboard; the scrape of a chair against hard flooring or a fork against a china plate; the screech of a heavy vehicle's brakes; an abrasive laugh; the whine of a mosquito; burps, slurps, chewings, and the noisy operation of other bodily functions. What is it that makes it the case that some things sound bad to us, and other things don't?

This enquiry is complicated by the fact that there is little philosophical consensus over what it is, exactly, that we hear when we make auditory contact with our surroundings. Options include sounds; ordinary objects such as bells, whistles, and fireworks (e.g. Pasnau 1999); and everyday events such as collisions and breakings (e.g. Casati et al. 2013; Leddington 2014). If we hear sounds, alone or in conjunction with objects or happenings, then we must address their ontological status: are they waves in an ambient medium (e.g. Meadows 2018); vibration events in or around material things (Casati and Dokic 1994); or sensible qualities like colours (e.g. Kulvicki 2008), for example?

A comprehensive examination of the aversive phenomenal character of unpleasant auditory experience would offer a systematic comparison of how each theory of the objects and contents of auditory perception aligns, or fails to align, with each candidate account of what gives a mental state its unpleasant phenomenology, and would determine which pair(s) of theories are jointly most plausible.

I will not attempt such an ambitious and exhaustive analysis here. Instead, I will consider how one prominent theory from the pain literature-the evaluativist view-might be transferred to the auditory domain. By focusing on evaluativism, we will see the terrain that any successful analysis of aversive auditory phenomenology must cover. In section two, I will explain which class of auditory phenomena will be the target of discussion, and why we should think of its members as relevantly similar to unpleasant bodily pains.

In section three, I outline the evaluativist account of unpleasant pain. In brief, evaluativism is a form of first order intentionalism about pain: it holds that pain experiences represent a state of bodily damage or disruption. ${ }^{3}$ Furthermore, unpleasant pains are said to represent this bodily condition evaluatively, as bad for the subject. In section four, I identify two problems that arise if we attempt to transfer evaluativism to the case of unpleasant auditory experience. The first

\footnotetext{
2 As, for example, with imperativist treatments that have aimed to unify (so far) pains, itches, and disgusting olfactory encounters (Hall 2008; Klein 2007; Martínez 2011, 2015) by identifying a common command-like content to each type of experience.

3 A content is first order if and only if it represents something in the non-mental world; a content is higher order if and only if it represents a mental state.
} 
problem is that evaluativism entails that an unpleasant auditory experience must represent some worldly phenomenon (an ordinary object of auditory perception), and to represent that phenomenon as bad for the listener. But the ordinary events and objects that generate awful noises very often afford no such threat to human agents. So the evaluativist must explain how and why we should accommodate this kind of systematic error within the evaluative content of auditory perception. The second problem is that the typical behavioural and cognitive reactions that a hearer exhibits in response to an awful noise appear to contradict the claim that an unpleasant auditory experience evaluates its object as bad: very often, the subject cares more about preventing or modulating the auditory experience itself, rather than about the sound or its source. In the concluding section, I sum up the constraints that must be met by any overarching account of unpleasant sensory affect, and indicate how the challenges raised against the evaluativist theory may apply to a wider range of positions in the pain literature.

Before we begin, notice that it is open to the evaluativist to deny that the theory is intended to encompass more than bodily pains, and so to argue that the auditory domain is outside of its purview. Perhaps these mental state types are sufficiently dissimilar that we should not expect to explain both in common terms. ${ }^{4}$ Nevertheless, the hypothesis that there is a property of unpleasantness that is capable of being shared by both bodily pains and auditory experiences is a plausible and productive starting point, and so it is worth examining. Moreover, its denial would leave the evaluativist with the burden of individuating multiple varieties of unpleasantness and explaining why evaluativism is restricted only to some of them. We can regard the argument of the paper as a conditional: if the evaluativist about unpleasant somatic pain intends for the theory to apply to a wider class of affective mental states, then it faces specific challenges in the auditory domain.

\section{Unpleasant auditory experience}

Our initial task is to delimit the category of auditory experience that will be the subject of analysis, and we can begin by setting aside certain undesirable episodes of hearing that are not our primary interest. Firstly, there are cases that seem to sit between the auditory and the physiological, such as the state we enter when we hear something excruciatingly loud, like the noise of a jet engine or an earthquake. The unpleasantness of an experience like this appears to reside in damage or distress at the ear, and so the evaluativist may simply be able to assimilate these states to bodily pains. I set these aside for present purposes not because the relationship between unpleasant pain in the sensory organs and unpleasant perceptual experiences delivered by those senses is uninteresting or unchallenging, but because there are core cases of unpleasant auditory phenomena that are not also instances of bodily pain.

\footnotetext{
4 This heterogeneity problem - that of identifying what qualitatively different affective experiences have in common-may apply even to different forms of unpleasant bodily pain, such as those caused by freezing and burning respectively (see, e.g., Clark 2005).
} 
Secondly, there are auditory experiences that are unwanted or to which we react negatively for purely extrinsic reasons: the ringing of a telephone when you are awaiting bad news; the alarm that marks the end of an exam you are struggling to complete on time; or distracting chit-chat during a lecture. We can set these aside because their undesirability derives from considerations that are extrinsic to the particular sound itself. Consider what you would report to a person who asks you why you find the sound disagreeable in each case. You would express your concern about what the alarm signals, what the ringing of the telephone portends, and the way in which the voices cause you to lose your focus. Each of these answers leaves room for an intelligible further question, namely whether there was something about the auditory experience itself that was unpleasant, ${ }^{5}$ the answer to which might be 'nothing at all'.

Compare these cases to a parallel instance of bodily pain. Suppose that a twinge in your knee signals the recurrence of an old, troublesome sporting injury; and that you know you now face months of physiotherapy and rehabilitation to return to full fitness. A friend asks you about the source of your anguish, and you report your disappointment and frustration about this lost time, and the efforts to come. This answer still leaves room for an intelligible further question, namely how bad the pain itself feels to you right now, the answer to which might be 'not bad at all'. The unpleasantness of the pain itself is thus not determined or exhausted by its significance for your wider projects and concerns.

Similarly, the auditory experiences of principal interest to the current discussion are those whose unpleasantness is not readily explained by appeal to extrinsic considerations. They can be found to be aversive by an agent whose task is simply to rate recorded sounds in order of unpleasantness, in controlled conditions, "without specific regard to their possible additional associative properties" (Kumar et al. 2008: 3812). ${ }^{6}$ Simply hearing fingernails being dragged down the surface of a chalkboard at a suitable volume, for example, is an unpleasant conscious state to enter; and likewise for exposure to microphone feedback, open-mouthed chewing, knuckle-cracking, and the myriad other noises we categorise as objectionable. Notice, too, that while the degree to which such auditory encounters are found to be unpleasant may differ from person to person (and, plausibly, from culture to culture), this is not to be explained in terms of differences in belief and desire. The fact that the stressful exam candidate finds the final bell disagreeable but the bored invigilator does not can be explained by appeal to these agents' differing beliefs about what the sound signifies for their projects and concerns, but this is not true for two people who differ in regard to how awful they find the sound of, say, a knife chafing against a glass bottle, in contexts where this sound bears no special practical significance for either party.

\footnotetext{
5 Notice that in each case the sound would be equally unwanted, feared, or disliked if we were to substitute it for any other with a similar acoustic profile. Indeed, the exam-alarm could be your favourite song, or your spouse saying "I love you", and still be something that you strongly desire not to hear.

${ }^{6}$ You can try this for yourself; recordings of the five sounds rated most highly for unpleasantness can be found here: https://soundcloud.com/wellcometrust/tracks.
} 
Unpleasant auditory states are like disagreeable bodily pains, and unlike affectively-neutral mental states such as beliefs, in that they have a motivational force of their own. Being in a conscious state of unpleasant pain motivates two kinds of action. ${ }^{7}$ Firstly and often most urgently, actions that are geared towards reducing or eradicating the pain itself (what Bain calls the self-eliminating character of pain (2019: 463)), such as taking an analgesic or soothing the skin. Secondly, actions that are geared towards treating the bodily disturbance that gives rise to the pain, such as seeking medical attention. The latter interventions have come to be known as 'first-order' actions, and they are directed at the non-mental world; while the former are 'second-order' actions, in being directed towards the phenomenal state of pain itself. Both kinds of motivation are defeasible, in that they may be outweighed by further considerations such as a desire to appear brave or a belief that taking painkillers is unhealthy.

Unpleasant bodily pains not only motivate but rationalise certain actions from the agent's own point of view. When we ask a subject why she lifted her hand from the scalding bathwater (Bain 2017: 42), she will report that she did so because of the pain it caused her. When we ask an agent why he took the aspirin, he will refer to the unpleasant headache he had been enduring; and so on. The reasons supplied by the agent are good reasons-we treat them as having settled the question of why the action was carried out, and as legitimate from the agent's perspective.

Unpleasant auditory experiences motivate their subject in two equivalent ways. They motivate second-order actions whose aim is to eliminate the experience itself (for instance, covering the ears); and they motivate first-order actions designed to eliminate the source of the experience (for instance, removing the scraping cutlery from the plate or asking a person to close their mouth while they eat). Again, it is the former class of actions that is the one that typically strikes the subject of experience with greatest urgency - the first thing one feels compelled to do upon encountering an awful noise is to limit one's auditory exposure to it, say by putting one's hands over one's ears, closing the window to muffle the outdoors, or turning down the volume of the radio. The motivations are defeasible in that the agent might take herself to have stronger reasons - such as reasons of politeness-not to curtail the sound at its source nor to prevent herself from hearing it.

Unpleasant auditory experiences not only motivate but rationalise certain actions, from the agent's own perspective. When we ask a subject why she covered her ears with her hands, it is perfectly intelligible for her to explain that it was because she found the squeal of the passing car's brakes unbearable. And when we ask someone why he switched off the radio, it is reasonable for him to reply that it was because the piercing tone of the singer's voice was so obnoxious.

In sum, there is reason to believe that there is a common unpleasantness shared by disagreeable bodily pains and awful auditory experiences, in spite of their other

\footnotetext{
7 See, especially, Bain (2017, Sect. 2.2), who calls the view that it is the pain itself that has motivational force 'motivationalism', and argues that it is widely accepted within the affect debate. For the purposes of this section, we can remain neutral on whether it is the pain itself, or some conjunct of the pain with a further conative or imperative state, that is motivating. The point is that an equivalent story will need to be told for the case of unpleasant auditory experiences.
} 
qualitative differences: both types of state have an aversive phenomenal character, and both provide reason for an agent to intervene upon the world and upon the conscious state itself. They are subjective states that we don't want to endure; that we seek to avoid or discontinue where possible. Both can be described using similar vocabulary-awful, unbearable, horrible, intolerable. We should thus consider it a goal of any candidate theory of affective phenomenology that it be able to capture the unpleasantness both of our responses to bodily damage and to awful noises, and to treat as flawed-or incomplete-any theory that lacks the resources to do so. In the following section, I outline the core tenets of the influential evaluativist theory of pain, in order to show in the remainder of the paper that it does not provide a satisfying analysis of the unpleasant phenomenology of disagreeable auditory experiences.

\section{The evaluativist theory of pain}

The evaluativist theory of unpleasant bodily pain (Bain 2013, 2017, 2019; Cutter and Tye 2011; Helm 2002) aims to explain the overall phenomenology of unpleasant pains, and to do so without violating the popular representationalist dictum that a mental state's felt character is exhausted by its intentional content. Evaluativism holds that unpleasant pain states have two distinct types of content:

(1) an indicative content that represents bodily damage

(2) an evaluative content that represents that bodily damage as bad for you ${ }^{8}$

Unpleasant pain states thus differ from ordinary perceptual states, on this view, in that they not only depict some state of affairs in the world, they deliver a negative appraisal of this state of affairs; that it is harmful, injurious, or otherwise threatening to one's interests. The indicative content of the pain explains its sensory phenomenology, including its shape and location, its being sharp or dull, and temporal qualities such as throbbing or stabbing. ${ }^{9}$ The evaluative content of the pain explains its unpleasantness: pains are unpleasant insofar as-and to the degree that - they evaluate the bodily disruption described in the state's indicative content as bad. Notice that the evaluative component of an unpleasant mental state is targeted at an element of the extra-mental world: it is the condition of a part of the body that is appraised as bad for the subject, not the mental state of pain itself. This makes evaluativism a first-order theory (it explains the unpleasantness of a mental state in terms of that state's representing something out in the world as bad), in contrast to second-order views that say for example that a conscious state is unpleasant when the subject desires that it be eliminated (e.g. Brady 2018), or when the state commands its own elimination (Barlassina and Hayward 2019). On a first-

\footnotetext{
8 See, e.g, Bain (2019: 464).

9 The sensory or indicative content of pain is interoceptive, describing an internal condition of the body. This type of content poses no special difficulty for standard psychosemantic theories. Cutter and Tye (2011), for example, offer a tracking account: pain states represent tissue damage because, under optimal conditions, they are tokened just when there is tissue damage.
} 
order view, a subject who is in pain is made to care about the extra-mental object of experience; on a second-order order view, the subject is made to care about her aversive mental state.

The evaluativist perspective yields a plausible analysis of the motivational structure of pain. Merely descriptive representational states such as beliefs are motivationally inert - they do not on their own give their subject any reason to act in one way or another. But a state that issues a negative evaluation of some bodily condition can provide a justifying reason for actions geared towards mitigating that condition; as when the pain state caused by my lifting a hot dish out of the oven motivates me to put the dish down quickly, by telling me that the damage it is causing to my hand is harmful for me.

The task of specifying the indicative component of bodily pain faces two related challenges (see Bain 2017). Firstly, there is the job of determining the nature of the bodily disturbance that is the characteristic object of this mental state-whether it be tissue damage (Cutter and Tye 2011); bodily disturbances in general (Bain 2017); the intensity of stimulation at the boundary of the skin (Gray 2019); or something else. Secondly, there is the job of ensuring that the indicative and evaluative contents of pain cohere with one another (Bain 2017:45): that is, that what is represented by the descriptive component of pain is the same as that which is represented by the state's evaluative content, and that it is a sufficiently plausible candidate for being evaluated as bad for the agent undergoing the experience. As Bain (op cit) points out, we ought not to say that my pain represents nociceptor activation, for example, because it is not plausible that nociceptor activation be represented as bad for me-because the firing of my pain receptors $i s n ' t$ bad for me. The indicative content of an unpleasant experience should specify some condition that $i s$ detrimental for me, such as harmful or threatening bodily damage.

As we will see in the following discussion, it is from a parallel line of thinking that problems arise for the evaluativist when transferring the theory from the domain of bodily pain to that of unpleasant auditory experience. That transfer faces the challenge of specifying an indicative content belonging to auditory perception that picks out something in the extra-mental world that is apt to be bad-harmful, damaging, threatening - for the subject in anything like the way that bodily injuries are bad. What the evaluativist must find, to preserve the parallel with unpleasant bodily pain, is an account of what is represented in auditory experiences of scraping fingernails; crying infants; squeaky brakes; episodes of sneezing and burping, and so on, such that what is indicated is also evaluated as bad for the listener. I will argue that evaluativism provides a credible model of only a narrow range of unpleasant auditory encounters, and that the approach must therefore concede that very many everyday experiences are illusory. Moreover, the ways in which agents respond behaviourally to an unpleasant auditory experience suggest that they care more about the perceptual state itself than about its object, contrary to the prediction of evaluativism. Before developing these lines of criticism, it will be helpful to briefly unpack the principal positions in the literature concerning what is perceived when we have an auditory experience. 


\section{Indication and evaluation in auditory perception}

All parties in the debate over the contents of auditory perception agree that ordinary veridical experiences in this modality arise when a wave is transmitted from a source event or object that is undergoing a process of vibration, through an elastic medium such as the air, to the ear. The different views on what we hear divide in their answers to two related questions: a) which stage of this causal process should be identified as the sound that is heard, and b) whether hearing (also) allows us to perceive the ordinary physical objects and events that are the sources of auditory experience. There are several ways of giving a positive answer to the second question, and this is significant for evaluativism because-being a first-order intentionalist view-it must locate something in the extra-mental world that is apt to be bad for human listeners.

On one kind of approach, we perceive the sources of sound indirectly, by hearing sounds directly. This is true, for example, on versions of the wave theory of sound, according to which sounds should be identified with the compression waves passing through the ambient medium, and acoustic qualities such as pitch, loudness, and timbre are treated as properties of those waves. ${ }^{10}$ An austere version of this view would hold that these waves are the only immediate objects of auditory perception, and that we infer the existence and nature of their sources much as we might infer that someone is home because their lights are on. On a richer view of auditory contents, however, the perception of compression waves mediates the perception of the sources of sound: we hear the cat indirectly by hearing the purr that she emits; we hear the breaking of the vase indirectly by hearing the crash that it generates, and so forth.

The indirect auditory perception of ordinary material phenomena is also permitted by certain distal theories of sound, which locate sounds in the vicinity of the reverberating objects that give rise to auditory experiences. Suppose we think that a sound is the event of the object vibrating (Casati and Dokic 1994) or the event of the object's setting the ambient medium into motion (O'Callaghan 2010), and that acoustic qualities are identical to or supervene upon these vibrations. On these views, to perceive a sound is not thereby to directly perceive a source object; it is to perceive something that the source is doing or undergoing. Hearing the vibration of the object allows us to hear the object indirectly: we hear the firework by hearing its percussive impact upon the surrounding air, for instance. The event of the object's vibrating can be considered as a part of a wider event that has further, non-audible qualities (O'Callaghan 2011), and this is consistent with saying that we hear the whole event by, or in virtue of, hearing that part of it which is audible.

On a second kind of approach, it is possible to hear source objects or events directly; that is, in a way that does not proceed by first perceiving a sound. This is

\footnotetext{
${ }^{10}$ Although this has some intuitive plausibility and a long history, especially in scientific thinking about sound, it is not a popular view among recent philosophers of auditory perception. A significant flaw of this view is that it entails that the spatial content of auditory experience is systematically in error: sound waves travel through the air at around 343 metres per second, but sounds are not perceived as doing sothey are perceived to be at, or coming from, their sources (see, e.g., O'Callaghan 2010).
} 
possible if we think, for example, that sounds are properties. For Kulvicki (2008), sounds are enduring dispositional properties of objects in much the same way that colours are: they are dispositions to react with a certain vibratory profile to the stimulation of being "thwacked". For Pasnau (1999), sounds are transient properties of an object, identical to or supervenient upon that object's vibrating over a time. On both of these accounts, ordinary objects are perceived directly in auditory experience when we hear these properties. Hearing the sound of the bell, construed as a perceptible quality of the bell, just is hearing the bell. Alternatively, sounds can be construed as properties of the events in which ordinary objects participate, such as episodes of colliding, breaking, or bouncing (Leddington 2014, 2019). This view permits of the direct auditory perception of such everyday events. Hearing the scrape of the turning key is sufficient for hearing the unlocking of the door. ${ }^{11}$ On a final version of the direct-perception approach, sounds are simply identified with ordinary events, as in the "fully Ockhamised" view of Casati et al. 2013, and not with their properties or component parts. Here, source events are perceived directly whenever we hear a sound, because they are the same thing. The clattering sound of the bottles falling over just is the event of the bottles falling over, and hearing the former is hearing the latter.

These accounts of the indicative contents of audition give the evaluativist some possible solutions to the question of how this modality might make us aware, either indirectly or directly, of things in our environment that might accurately be represented as bad for us. The first kind of approach enables the evaluativist to say something like the following: a person hears the whine of a mosquito, construed as a compression wave in the air or the event of the insect's wings disturbing the air, and in virtue of doing so also hears the mosquito itself. The auditory experience is unpleasant in virtue of its evaluating the mosquito as bad for the subject. Or on the second, more immediate approach, the evaluativist might state that a subject hears the sound of a wet, hacking cough, construed as either a part or a property of that event, and in doing so hears that event directly. The subject's auditory experience is unpleasant in virtue of representing that coughing event as bad for her. In scenarios like these, we can see how both the indicative and the evaluative contents of the auditory experience might be considered to be accurate. There is a mosquito, and mosquitoes tend to be harmful for humans because they bite and transmit disease. There is a cough, and coughs can be bad for humans because they spread contagion. The same would plausibly be true of objects like wasps, snakes, and dentists' drills; and of events like vomiting, spitting, and screaming. So far so good for evaluativism about unpleasant auditory experience. ${ }^{12}$

\footnotetext{
11 "We hear sounds directly, but they do not 'mediate' between us and their event sources. Instead, just as we see objects in (but not in virtue of) seeing their colours, so we hear event sources in (but not in virtue of) hearing their sounds." (Leddington, 2019:6, original emphasis).

12 Notice that this kind of account is not available to an evaluativist who takes a more austere perspective on the indicative contents of auditory perception. If all that is heard is airborne compression waves, for example, then the evaluative content of an unpleasant auditory experience would almost always be false-with very few exceptions, such as sounds that are so loud they knock us off our feet, waves in the air are never bad for human agents. If all that is heard is an event of vibration, the same point can be made. The mere activity of reverberation does not make an object into a threat for human listeners. These
} 


\subsection{Sounds and innocuous sources}

The problems for evaluativism begin to arise when we turn our attention to the multiplicity of unpleasant auditory experiences that are not caused by material things and events that pose any danger of harm to us; what we can call innocuous cases. Squeaky gates; mechanical brakes; nasal laughter; the squeals of excitable toddlers; yowling tom-cats; microphone feedback; poorly-tuned violins; fingernails running down a chalkboard; cutlery against crockery; electric band-saws; buzzing refrigerators; open-mouthed snoring; vacuum cleaning; several children playing the recorder together; baby seagulls; discordant singing; polystyrene being rubbed against itself: these things and many others like them are routine causes of unpleasant auditory states, but none of them constitute any credible threat for human listeners and their wellbeing. For evaluativism to accommodate the unpleasantness of auditory encounters with these innocuous sorts of entities and happeningswhich, I would hazard, generate the vast majority of aversive auditory phenomenology - it must treat the relevant evaluative contents as being guilty of widespread error. It must say, that is, that while the indicative content of auditory experience may be largely accurate in its depiction of what is going on in our surroundings, the evaluative component of unpleasant auditory states is quite systematically mistaken in its appraisal of the badness of those goings on. An errortheory of this nature would represent a significant departure from the evaluativist position on unpleasant somatic pain, which holds that painful experiences are predominantly accurate in their evaluation of bodily damage as bad for the subject.

The second problem for evaluativism about unpleasant auditory experience is that it can be shown that what the subject of experience is made to care about in undergoing a conscious episode of this sort is not the extra-mental source object or event that is the supposed target of a negative evaluation at all. It is the sound that is associated with that source, and it is the hearing of that sound. When we consider more closely what it is that unpleasant auditory experiences motivate their subject to do, we see that they stimulate actions whose aim is to adjust or eliminate the audible qualities associated with the source and actions whose aim is to prevent the subject from having an experience of those qualities. Why, if the perceptual state evaluates the source as bad or harmful for the agent, should it be rational for her to act in these ways?

Consider the following two everyday cases in which your auditory experience motivates a first-order (world-directed) action:

i. Whistling Cooker. The gas ring on your cooker emits a thin, high-pitched whistle when it is alight and the dial is set at a particular angle. The phenomenal state that you enter when you hear the whistle is unpleasant, and you adjust the dial by a fraction until the tone is more bearable.

Footnote 12 continued

considerations should lead the evaluativist towards a richer account of auditory content that permits of the perception of ordinary, 'interesting' events. 
ii. Chalkboard Nails. I drag my fingernails down the surface of the chalkboard, generating a characteristically abrasive scraping noise. Your auditory experience is unpleasant, and you ask me to stop.

In both scenarios, there is nothing in the extra-mental world that poses a threat to your interests, and your behavioural responses reflect this. Suppose we ask why you acted as you did, and whether it was rational for you to do so. In whistling cooker, it would be perfectly intelligible for you to reply that you turned the dial in order to alter the character of the sound it was making, because it was unpleasant in virtue of its pitch and tone. Your concern for the source event begins and ends with the whistling noise (whether this is a matter of its producing a compression wave; vibrating in a particular manner; or having some other audible feature), and your action reflects this concern: you are content to leave the event itself essentially unchanged provided it no longer whistles. In chalkboard nails, too, it is the stridulous sound of the event that gives you a reason for this first-order action. You care about the source event only insofar as it is a sound-maker. If in each case your auditory state evaluates the source event as bad for you, it is not clear why you should be so concerned about these events' audible qualities.

Consider the following third case, in which you are motivated to perform a second-order (state-directed) action:

iii. Braking Train. Your train arrives alongside the platform, and it slows with a prolonged metallic squeal of brakes. You clamp your hands over your ears until it comes to a halt.

Here your action is designed to eliminate the auditory experience of the braking train rather than to intervene upon the extra-mental world. This introduces an auditory variant of the messenger-shooting objection that has been raised against evaluativism about pain (Jacobson 2013, 2019; Bain 2013). If a pain state really informs the subject of a harmful bodily condition, this challenge proceeds, then it is not clear why it should motivate and rationalise self-eliminating behaviour. Acting to stop a pain seems just as irrational as shooting a messenger who brings bad news. In the auditory case, the worry is the same: if an unpleasant experience of a sound is in the business of evaluating threats and harms out there in the world, why should it be rational for the hearer to prevent herself from having such experiences? Blocking out the sound appears just as irrational as the behaviour of a child who sticks his fingers in his ears to avoid unwelcome news. ${ }^{13}$ If your auditory experience of the slowing of the train represents that event as bad for you, it isn't clear why this

\footnotetext{
13 Notice that this case has a secondary function in my argument: it applies even if the evaluativist adopts a fully-Ockhamised account of sounds. In response to cases (i) and (ii), a proponent of that view might argue that there is no real separation between the event and the sound it is said to produce, so it is not intelligible to speak of actions that are designed to affect the sound of the event rather than the event itself. If there's no ontological gap between the whistling and the burning cooker ring, for instance, then we cannot reasonably speak of actions that alter the whistling without altering the burning cooker ring. The lesson of braking train remains even if hearing the squeal is hearing the train; because your secondorder action still shows no regard for that event, independently of your experience of it.
} 
should motivate you to block your perceptual access to this event. Returning to whistling cooker and chalkboard nails, we can see that the so-called first-order actions in these cases are really second-order actions in disguise. You intervene on parts of the extra-mental world only because of the auditory experiences they give rise to: you turn the dial to eliminate your experience of the whistling, and you ask me to remove my nails from the board because of how it sounds to you.

Notice how different these situations are from those in which you feel an unpleasant bodily pain and your first-order actions have as their aim the amelioration of an extra-mental condition of damage that is bad for you, independently of the aversive experience to which it gives rise. The victim of unpleasant pain is motivated to attend to the site of this damage because it is a condition that is harmful for them. In the innocuous auditory cases, by contrast, the subject has no independent reason to intervene upon the world and does not take herself to have such a reason. The three scenarios put pressure on the idea that auditory experiences evaluate source events as bad for the listener, by showing that they motivate actions whose principal aim is to eradicate the subject's own experiential states.

A second set of problem cases illustrates how difficult it is to identify just which sound source might be the object of a negative perceptual evaluation.

Consider the following two examples:

iv. Acousmatic Listening. Those who are musically trained are often able, in what is known as acousmatic listening, to "spontaneously detach [a] sound from the circumstances of its production, and attend to it as it is in itself" (Scruton 1997: $2-3)^{14}$

The auditory attention of the acousmatic listener is directed to the sonic qualities of sounds alone, divorced from their sources - they are not heard as products of musical playing, for example, or of other ordinary happenings in the material world. They are listened to for their tone and pitch; melody; purity; dynamics; timbre; loudness; rhythm; etc., and the listener may gain no insight at all into the nature of the source event, and remain unable to identify the origin of the sounds she experiences. ${ }^{15}$ When acousmatic listening yields an unpleasant auditory experience, then, it is not clear which particular event in the subject's surroundings might be evaluated as harmful or bad for the subject. The problem for the evaluativist comes with specifying the ordinary event that is to be negatively appraised, when that event is not a focal part of the indicative content of experience.

v. Tape Recording. You put on headphones, and listen to a selection of unfamiliar electronic buzzes and bleeps, some of which are unpleasant to hear. The sounds appear to you to be occurring within your own head.

\footnotetext{
14 The notion is not uncontroversial. For critical discussion, see e.g. Hamilton (2009).

15 In Scruton's terms, sounds "float free from their causes" and are heard "apart from the material world" (p. 221).
} 
Evaluativism entails that the source of these sounds is represented as bad for you; that there is some unknown state of affairs inside your skull that is perceived to pose a threat to your interests. Once again, it is not clear how this source is represented in your experience, except as the origin of the sounds that you hear; and it's not clear what kind of badness might be attributed to this source independently of its effect on your auditory phenomenology. It follows that it is similarly unclear which first-order actions the subject of experience might be motivated to perform on the basis of this evaluative content. Similar puzzles apply in scenarios where the sound in your unpleasant auditory encounter is perceived to be bouncing around a space, or filling it, or arriving from an indeterminate location; or where the sound outlasts its source. These cases indicate the difficulties associated with pinning down the ordinary material events and entities that are said to be the objects of a negative evaluation in unpleasant auditory experience.

\subsection{Error and evaluation}

Let us return to cases where the unpleasant auditory experience is not mistaken in its evaluation of some worldly state of affairs as bad, such as the whine of a biting insect or the noxious sound of vomiting. Not all of the considerations raised in the previous section apply to these scenarios. For example, it is not obvious that is rational for a person to cover her ears when she hears a mosquito buzzing nearby. What is justified, instead, is killing the mosquito or releasing it from the room. And it isn't evident that the most rational course of action when someone is vomiting close to you is to prevent yourself from hearing this event. Better, instead, to leave the vicinity. So the messenger-shooting objection lacks some of its usual force in cases like these, and the claim that the auditory experience represents the source event as bad for the subject is more credible. ${ }^{16}$ Similarly, these situations do not involve acousmatic listening, and nor do the sounds in question have indeterminate or unlocatable sources.

So the evaluativist might maintain that unpleasant auditory experiences $d o$ bear a first-order evaluative content, and hold that their position is vindicated by paradigm scenarios in which the evaluative content accurately picks out some threatening state of affairs in the non-mental world. Unpleasant auditory experiences with innocuous sources, meanwhile, might be explained in terms of relevant audible similarities to the paradigm cases: they represent harmless sources as bad because of their perceived similarity to harmful sources. This would be to embrace the errortheory when it comes to the innocuous cases, conceding that the evaluative content of experience gets things wrong in many everyday contexts, but to argue that the propensity for error can nonetheless be accounted for (perhaps in evolutionary terms, as with psychologists of audition who have explored the hypothesis that we find the scraping of a chalkboard unpleasant because it is acoustically similar to monkey distress calls (e.g. Halpern et al. 1986; McDermott and Hauser 2004)).

\footnotetext{
16 This is, I take it, to be charitable to the evaluativist. It surely is often reasonable, from the agent's own point of view, to eliminate an unpleasant auditory state even when it accurately evaluates a potential harm.
} 
To pursue this kind of error-theoretic solution, the evaluativist must argue that there is something in common between relevant types of harmful and innocuous event that explains why both are evaluated as bad when they are heard. How this might be done depends upon which theory of auditory perception is adopted. On a wave theory of sound, for example, it might be possible to locate a salient similarity in the sonic waves that each event produces-to say, for instance, that both screeching predators and chalkboard nail-scraping generate similar wave patterns in the surrounding air. Then there might be an evolutionary story to tell about how and why human listeners come to respond with negative evaluative auditory states when they encounter sufficiently similar wave patterns. If, instead, the immediate objects of auditory perception are vibration events in objects then it might be held that we have evolved to evaluate certain vibration events as bad for us, whether the vibrating entities are harmful or not. And if we hear ordinary events or objects directly, then the error-theorist will need to identify something in common among such diverse phenomena as monkey calls, dentists' drills, and microphone feedback, in order to explain why we perceptually evaluate all of them as bad for us, despite their many dissimilarities.

In filling out one or other of these options, two challenges remain for the evaluativist. Firstly, there is the challenge of specifying which actions it is rational for an agent to perform when she undergoes an unpleasant auditory experience in the harmful and innocuous cases respectively, given that the evaluative content is accurate in the former but erroneous in the latter. For the evaluativist, all unpleasant auditory experiences represent their objects as bad for the listener, and so all such states provide defeasible motivation for first-order actions designed to eliminate those objects or to put distance between them and the subject. When an unpleasant auditory experience correctly evaluates some state of affairs as bad for you, then it makes it rational for you to intervene in order to alleviate that state of affairs-like when your auditory state motivates you to kill the mosquito. But scenarios like those introduced in the previous section indicate that when the sound-source is innocuous and the evaluative content of your experience is illusory, it seems rational from your point of view to eradicate the auditory state itself, perhaps by covering your ears, and to leave the world otherwise untouched. The experience has a self-eliminating motivational force, rather than galvanising first-order behaviours. Now the burden for the evaluativist is to explain what it is that underpins this difference: what it is that makes it reasonable from the agent's perspective to act upon the extra-mental world in the first type of case, and upon her own experiential state in the second. One option is to say that the subject has a belief that the sound-source is not harmful or otherwise bad in the innocuous cases, and this outweighs any reason presented by the experience itself to perform a first-order action. For instance, the agent believes that the braking train presents no threat to her interests, despite what the evaluative component of her auditory experience is telling her, and this explains why she is concerned only with eliminating her auditory experience of the train. Whether this kind of explanation is tenable will rest upon how the evaluativist conceives of the interplay between cognitive and perceptual states in such cases; the temporal order in which they arise; whether they always participate in an explicit process of reasoning; and so forth. But it is not at all obvious that such a conflict between the 
content of one's perceptual and doxastic states is reflected in the phenomenology of auditory encounters like the braking train case.

Secondly, there is a question of whether the indicative and evaluative contents of auditory perception can still be made to cohere in the way that Bain (2017) has argued is necessary for a successful evaluativist treatment of unpleasant mental states. Recall that the challenge is to ensure that what is evaluated by a perceptual state is the same thing that the state indicates in its descriptive content. For somatic pain, this is satisfied by saying that it is bodily damage that is both indicated and evaluated. For auditory perception, the situation is not so simple. Consider again that the evaluativist must find some common indicative content among unpleasant auditory experiences of both harmful and innocuous sources, in order to substantiate the claim that (for example) we find hearing car alarms unpleasant because they sound similar to the calls of predators from our evolutionary past. The only plausible commonality between such cases is that they give rise to phenomenally similar auditory experiences, whose indicative contents pick out similar acoustic qualities such as loudness, pitch, timbre, and tone. But this appears to reveal a lack of coherence between the indicative and evaluative contents of unpleasant auditory states: the indicative content describes a particular pattern of acoustic qualities, but it is surely not the case that the state evaluates those very same qualities as bad for the listener. After all, it is not in virtue of their pitch and timbre that threats to our interests are bad for us - they are bad because they bite us, make us ill, prey on, eat, or otherwise injure us. So the task for the evaluativist is to ensure a match between what is indicated and what is evaluated in auditory experience, so that both elements of an unpleasant auditory state are directed at a common phenomenon.

\section{Conclusion}

Can the conceptual resources of the evaluativist theory of unpleasant bodily pain be transferred neatly to the domain of unpleasant auditory experience? I have argued that a central concern for this project is that of identifying a suitable indicative content of auditory representations, such that what is depicted is also a plausible candidate for negative evaluation. There are theories of auditory perception that permit that we can hear everyday objects and events-things colliding, bouncing, scraping, breaking, and so forth-and these allow the evaluativist to maintain that unpleasant auditory experiences are those that evaluate certain environmental goings-on as bad for the subject. But the evaluative component of an unpleasant auditory state would be accurate only in a rather narrow range of cases: those where what sounds awful is independently awful for the listener. In all other scenarios, including paradigm terrible sounds such as the scrape of nails down a chalkboard, the evaluativist is led to an error-theory of auditory perception's evaluative content. Fingernails and chalkboards just aren't bad for us, except insofar as they generate aversive phenomenology. Furthermore it is unclear, given the sorts of experiences surveyed in Sect. 4.1, that unpleasant auditory encounters with innocuous sources of sound really do involve a negative evaluation of those sources. The listener shows little inclination to act upon those sources except to alter their audible properties; 
cannot always perceptually locate or identify those sources; and exhibits an overarching concern for the elimination of her own conscious auditory state.

I close with two sets of brief remarks concerning the wider significance of the arguments raised in the paper for debates concerning affective phenomenology. Firstly, the reader might by now be more persuaded that one who holds evaluativism about unpleasant bodily pain has no real obligation to commit also to evaluativism about auditory unpleasantness, given how different these mental state types are. In which case, arguments that attempt to block the transfer of evaluativism from one domain to the other will have little purchase. Notice, however, that this strategy would commit the pain-evaluativist to a disunified treatment of the aversive phenomenal character of unpleasant experience; a theoretical disunity that many philosophers of mind will be tempted to avoid. Under representationalism, the idea would be that states of somatic pain are unpleasant in virtue of their evaluative content, while obnoxious auditory states (plus, perhaps, offensive olfactory experiences; foul tastes, and so on) get their unpleasantness from another, as-yet unidentified source. Pain is one thing; sensory affect is another. While this disjunctive approach is certainly not impossible, the absence of a common theoretical analysis of unpleasant phenomenal character is dissatisfying for those who value parsimony. Many authors in the pain literature speak in general terms of "affective phenomenal character" or "unpleasant sensory experience" as a single explanandum (e.g. Barlassina and Hayward 2019; Aydede and Fulkerson 2019), so to restrict evaluativism to the case of unpleasant somatic pain is to concede a certain theoretical narrowness.

Secondly, although my primary aim has been to raise a specific challenge for evaluativism about unpleasant auditory experience, we can see how the considerations raised above may be applied to a wider family of approaches from the pain literature. The scenarios in Sect. 4.1 indicate that experiences of awful noises tend to generate and justify second-order, state-directed actions (such as covering the ears), contrary to the predictions of the evaluativist view. But other first-order theories of affective phenomenology may make the same erroneous predictions. For instance, consider a first-order imperativist treatment (e.g. Martínez 2011, 2015) according to which states of bodily pain issue commands such as see to it that this bodily disturbance is no more! (Martínez 2011: 78) or cease acting in a way that would cause more injury! (Klein 2007: 520). When translated to the case of hearing sounds, first-order imperativism would have to assert that unpleasant auditory states also issue a command to the subject of experience, directing them to do something about the extra-mental world. Again, the task for this theory would be to defend an ontology of sounds that permits us to identify what it is in the non-mental environment that is the target of such behavioural interventions-surely not pressure waves in the air! - and to pinpoint a plausible set of first-order actions that are commanded when the subject undergoes an unpleasant auditory experience.

A more palatable, unifying alternative may be found in second-order theories of sensory affect. Perhaps unpleasant auditory states generate, or are partly constituted by, an occurrent desire for the state to end (following Brady's (2018) higher-order desire theory of pain); perhaps unpleasant auditory states are accompanied by a higher-order directive representation that commands their cessation (following 
Klein's (2015) second-order imperative account); or perhaps they command their own elimination (as Barlassina and Hayward's (2019) reflexive imperativism would have it). I take no stand here on which of these options, if any, is best equipped to accommodate the affective phenomenology that arises when we encounter awful noises, but hope instead to have laid some of the conceptual groundwork for future research on the topic.

Acknowledgements I would like to thank Andrew Buskell, Adrian Currie, Søren Overgaard, and an anonymous reviewer for this journal for their very helpful critical feedback on earlier drafts of this paper.

Open Access This article is licensed under a Creative Commons Attribution 4.0 International License, which permits use, sharing, adaptation, distribution and reproduction in any medium or format, as long as you give appropriate credit to the original author(s) and the source, provide a link to the Creative Commons licence, and indicate if changes were made. The images or other third party material in this article are included in the article's Creative Commons licence, unless indicated otherwise in a credit line to the material. If material is not included in the article's Creative Commons licence and your intended use is not permitted by statutory regulation or exceeds the permitted use, you will need to obtain permission directly from the copyright holder. To view a copy of this licence, visit http:// creativecommons.org/licenses/by/4.0/.

\section{References}

Aydede, M. (2009). Is feeling pain the perception of something? Journal of Philosophy, 106(10), 531-567.

Aydede, M., \& Fulkerson, M. (2019). Reasons and theories of sensory affect. In D. Bain, M. Brady, \& J. Corns (Eds.), The philosophy of pain: Unpleasantness, emotion, and deviance. New York: Routledge.

Bain, D. (2013). What makes pains unpleasant? Philosophical Studies, 166(1), 69-89.

Bain, D. (2017). Evaluativist accounts of pain's unpleasantness. In J. Corns (Ed.), The Routledge handbook of the philosophy of pain (pp. 4-50). London: Routledge.

Bain, D. (2019). Why take painkillers? Nous, 53(2), 462-490.

Barlassina, L., \& Hayward, M. K. (2019). More of Me! Less of Me!: Reflexive imperativism about affective phenomenal character. Mind, 128(512), 1013-1044.

Brady, M. S. (2018). Painfulness, desire, and the euthyphro dilemma. American Philosophical Quarterly, 55(3), 239-250.

Casati, R., Di Bona, E., \& Dokic, J. (2013). The ockhamisation of the event sources of sound. Analysis, 73, 462-466.

Casati, R., \& Dokic, J. (1994). La Philosophie du Son. Nîmes: Chambon.

Clark, A. (2005). Painfulness is not a Quale. In M. Aydede (Ed.), Pain: New essays in its nature and the methodology of its study. Cambridge: MIT Press.

Corns, J. (2014). Unpleasantness, motivational oomph, and painfulness. Mind and Language, 29(2), $238-254$.

Corns, J. (2018). Recent work on pain. Analysis, 78(4), 737-753.

Cutter, B., \& Tye, M. (2011). Tracking representationalism and the painfulness of pain. Philosophical Issues, 21(1), 90-109.

Gray, R. (2019). On the content and character of pain experience. Pacific Philosophical Quarterly, $100(1), 47-68$.

Hall, R. J. (2008). If it itches, scratch! Australasian Journal of Philosophy, 86(4), 525-535.

Halpern, D. L., Blake, R., \& Hillenbrand, J. (1986). Psychoacoustics of a chilling sound. Attention, Perception, \& Psychophysics, 39(3), 77-80.

Hamilton, A. (2009). The sound of music. In M. Nudds \& C. O'Callaghan (Eds.), Sounds and perception: New philosophical essays. Oxford: Oxford University Press. 
Helm, B. (2002). Felt evaluations: A theory of pleasure and pain. American Philosophical Quarterly, 39(1), 13-30.

Jacobson, H. (2013). Killing the messenger: Representationalism and the painfulness of pain. The Philosophical Quarterly, 63(252), 509-519.

Jacobson, H. (2019). Not only a messenger: Towards an attitudinal-representational theory of pain. Philosophy and Phenomenological Research, 99(2), 382-408.

Klein, C. (2007). An imperative theory of pain. Journal of Philosophy, 104(1), 517-532.

Klein, C. (2015). What pain asymbolia really shows. Mind, 124(494), 493-516.

Kulvicki, J. (2008). The nature of noise. Philosophers' Imprint, 8, 1-16.

Kumar, S., Forster, H. M., Bailey, P., \& Griffiths, T. D. (2008). Mapping unpleasantness of sounds to their auditory representation. Journal of the Acoustical Society of America, 124(6), 3810-3817.

Leddington, J. (2014). What we hear. In R. Brown (Ed.), Consciousness inside and out: Phenomenology, neuroscience, and the nature of experience (pp. 321-324). Dordrecht: Springer.

Leddington, J. (2019). Sounds fully simplified. Analysis, 79, 621-629.

Martínez, M. (2011). Imperative content and the painfulness of pain. Phenomenology and the Cognitive Sciences, 10(1), 67-90.

Martínez, M. (2015). Disgusting smells and imperativism. Journal of Consciousness Studies, 22(5-6), 191-200.

McDermott, J., \& Hauser, M. (2004). Are consonant intervals music to their ears? Spontaneous acoustic preferences in a nonhuman primate. Cognition, 94(2), B11-B21.

Meadows, P. J. (2018). In defense of medial theories of sound. American Philosophical Quarterly, 55(3), 293-302.

O'Callaghan, C. (2010). Constructing a theory of sounds. Oxford Studies in Metaphysics, 5, 247-270.

O'Callaghan, C. (2011). Hearing properties, effects, or parts? Proceedings of the Aristotelian Society, $111(3 \mathrm{pt} 3), 375-405$.

Pasnau, R. (1999). What is sound? The Philosophical Quarterly, 49(196), 309-324.

Scruton, R. (1997). The aesthetics of music. Oxford: Clarendon Press.

Publisher's Note Springer Nature remains neutral with regard to jurisdictional claims in published maps and institutional affiliations. 